\title{
On Sinaĭ Billiards on Flat Surfaces with Horns
}

\author{
Henk Bruin ${ }^{1}$
}

Received: 19 May 2020 / Accepted: 24 March 2021 / Published online: 20 April 2021

(c) The Author(s) 2021

\begin{abstract}
We show that certain billiard flows on planar billiard tables with horns can be modeled as suspension flows over Young towers (Ann. Math. 147:585-650, 1998) with exponential tails. This implies exponential decay of correlations for the billiard map. Because the height function of the suspension flow itself is polynomial when the horns are Torricelli-like trumpets, one can derive Limit Laws for the billiard flow, including Stable Limits if the parameter of the Torricelli trumpet is chosen in $(1,2)$.
\end{abstract}

Keywords Billiards $\cdot$ Suspension flow $\cdot$ Limit laws $\cdot$ Decay of correlations

Mathematics Subject Classification Primary: 37D50 · Secondary: 37A25 · 37A60

\section{Introduction}

Recent results on the statistical properties of non-uniformly hyperbolic flows in dynamical systems include polynomial mixing rate, when the flow can be modeled as suspension flow over a Gibbs-Markov map or over a Young tower, and the roof function $h$ of the suspension flow has polynomial tails:

$$
\mu(\{x \in M: h(x)>t\})=\ell(t) t^{-\beta},
$$

where $\ell(t)$ is a slowly varying function. There are geodesics flows [1] on non-compact surfaces of curvature -1 where the above tail condition with $\beta=1$ or 2 applies (although properties of Kleinian groups rather than Young towers are used in the modeling). For Lorentz gas with infinite horizon, the parameter in (1) is $\beta=2$. So, although the theory puts no restriction on the parameter $\beta$ in (1), these examples provide us only with very specific values of $\beta$. The model of [13] based on two convex scatterers with points of zero curvature directly opposite to each other produces finite measure cases with variable $\beta>1$ for the roof function of the Young tower (but without giving the slowly varying function in (1)). In dimension one, the Pomeau-Manneville maps allow for inducing schemes where the induce

Communicated by Alessandro Giuliani.

$凶$ Henk Bruin

henk.bruin@univie.ac.at

1 Faculty of Mathematics, University of Vienna, Oskar Morgensternplatz 1, 1090 Vienna, Austria 
time satisfies (1) for $\beta=1 / \alpha$ where $\alpha$ is the order of contact between the graph and the tangent at the neutral fixed point. Thus $\beta>0$ can be chosen freely, but despite some higherdimensional variants, Pomeau-Manneville maps remain too specific to play a substantial role in the modeling of billiards or other mechanical models. In [7,20,22,23,27] it was shown that almost Anosov diffeomorphisms (and flows [8]) also allow inducing schemes with tails satisfying (1). These are non-uniformly hyperbolic invertible systems, and, in contrast to Pomeau-Manneville maps, can be chosen to be $C^{\infty}$ or real analytic, even if $\beta$ is non-integer.

The purpose of this paper is to provide a class of examples that fit directly in the context of billiard maps, and which can be modeled by suspension flows over Young towers with tails as in (1). The basic ingredient is the geodesic flow on a surface of revolution, which we call horns, of which the Torricelli trumpet is an example. New (or at least we are not aware of explicit calculations in the literature) is a one-parameter family of Torricelli trumpets, which provide tails as in (1) where the exponent $\beta$ is equal to the parameter of the family.

Let the billiard table $\mathcal{Q}$ be a flat compact manifold, such as a torus or a rectangle with reflecting boundaries. We assume that

- there are finitely many circular horns and/or scatterers $H_{i}, i=1, \ldots, N$, of radius $r_{i}$ (so of curvature $\left.1 / r_{i}\right)$;

- their closures are disjoint closures, so the minimal flight time between collisions $\tau_{\min }>0$;

- the horizon is finite, i.e., the maximal flight time $\tau_{\max }$ between collisions is finite.

- Scatterers are "hard balls", i.e., the collision rule of the particle with such scatterers is the rule of fully elastic reflection.

- Horns act as "soft balls", in the sense that they reflect the particle, but not according to the law of elastic collision: although the angle of incidence equals the angle of reflection (up to a minus sign: $\varphi^{+}=-\varphi^{-}$), the entrance position on $\partial H_{i}$ is not necessarily the exit position.

A unit mass, unit speed particle moves on this surface with scatterers. It reflects fully elastically at the scatterers, but when it meets a horn $H_{i}$, it moves up on the surface of the horn, keeping its speed but observing the law of preservation of angular momentum and the holonomic constraint keeping it in $H_{i}$, until it exits $H_{i}$ again and resumes its trajectory on $\mathcal{Q}$. The excursion time is $2 t_{\max }\left(\varphi^{+}\right)$where $\varphi^{+} \in\left[-\frac{\pi}{2}, \frac{\pi}{2}\right]$ is the angle of incidence that the particles trajectory makes with the normal vector to $\partial H_{i}$, and $t_{\max }$ is the time for an excursion to reach the highest point in the horn. Due to the radial symmetry of $H_{i}$, the angle of incidence $\varphi^{-}=-\varphi^{+}$.

We denote the flow on $\mathcal{Q} \cup \bigcup_{i=1}^{N} H_{i}$ by $\phi^{t}$. The excursions of the particle on the horn can take an unbounded amount of time, so that, despite the bounded distance between, the flow-time between incoming collisions can be unbounded.

Let us parametrize the circle $\partial H_{j}$ by the position $\theta^{-}$(measured clockwise as an angle in $[0,2 \pi)$ but in order to avoid confusion with the angle of incidence/reflection, we will refer to $\theta^{-}$as the position). The exit position $\theta^{+}$is a function of the entrance position $\theta^{-}$and the angle of reflection $\varphi^{+}$. The rotation function ${ }^{1}$

$$
\Delta \theta:=\theta^{+}-\theta^{-}
$$

depends on $\varphi^{+}$but (due to radial symmetry) not on $\theta^{-}$. We prefer to let $\Delta \theta$ depend on the outgoing angle $\varphi^{+}$, in order to follow the conditions of Bálint \& Tóth [5,6], see Sect. 2.1. Note that $\Delta \theta=0$ at scatterers, and at horns for $\varphi^{+}= \pm \frac{\pi}{2}$, i.e., grazing collisions. Since

\footnotetext{
1 using the terminology in [5].
} 
$\varphi^{+}=-\varphi^{-}$, the resulting reflection map

$$
R: M^{-} \rightarrow M^{+}, \quad\left(\theta^{-}, \varphi^{-}\right) \mapsto\left(\theta^{+}, \varphi^{+}\right)=\left(\theta^{-}+\Delta \theta\left(-\varphi^{-}\right),-\varphi^{-}\right)
$$

represents the outgoing position and angle as function of the incoming position and angle. Here $M^{ \pm}=\cup_{i=1}^{N} M_{i}^{ \pm}$for $M_{i}^{ \pm}=\partial H_{i} \times\left[-\frac{\pi}{2}, \frac{\pi}{2}\right]$ are the incoming and outgoing phase spaces, copies of one another, but formally not the same.

The flight map $F: M^{+} \rightarrow M^{-}$is given by

$$
\left(\theta_{i}^{+}, \varphi_{i}^{+}\right) \in M_{i}^{+} \mapsto\left(\theta_{j}^{-}, \varphi_{j}^{-}\right)=\left(\theta_{j}^{-}, \pi+\varphi_{j}^{+}+\theta_{j}^{-}-\theta_{i}^{+}\right) \in M_{j}^{-},
$$

where $i, j$ are the indices of the scatterers or horns of the consecutive collisions. The composition $T=R \circ F: M^{+} \rightarrow M^{+}$is the billiard map, expressed in outgoing coordinates from one horn or scatterer to the next. Note that $T$ has singularities at $\cup_{i=1}^{N} \partial H_{i} \times\left\{ \pm \frac{\pi}{2}\right\}$ and at $\cup_{\text {horns }} H_{i} \partial H_{i} \times\{0\}$ (non-compactness of horns).

It is worth comparing the collision with horns to scatterers with finite range potentials $V$, as considered in e.g. [3,17,18,24-26]. Both are modeled by the formula (2) where in case of finite range, radially symmetric, potentials $V$ and energy level $E$,

$$
\Delta \theta=2 \int_{r_{\min }}^{r_{i}} \frac{r_{i} \sin \varphi d r}{\sqrt{r^{2}(1-2 V(r))-r_{i}^{2} \sin ^{2} \varphi}},
$$

see [18, Formula (3.7))] or [5, Formula (5.2)] for the energy level $E=\frac{1}{2}$ of a unit mass. An important quantity is the derivative of the rotation function $\Delta \theta$ :

$$
\kappa(\varphi):=\frac{d}{d \varphi} \Delta \theta(\varphi) \quad \text { for } \varphi=\varphi^{+} .
$$

In [18] it is shown that the billiard flow is hyperbolic and ergodic if the range of $\kappa$ is disjoint ${ }^{2}$ from $[-2,-2+\delta]$ for some $\delta>0$. As shown in [17, Proposition 5.2], if the scatterer has a smooth finite range potential, then $\lim _{\varphi \rightarrow \pm \frac{\pi}{2}} \kappa(\varphi)=-2$, and indeed, there are several results showing that the ergodicity of the billiard map can fail, cf. [3,17,24,26,30]. It is not clear, however, that our horns can be modeled as scatterers with finite range potentials. For instance, sojourn times in the horn (and hence $\Delta \theta$ ) are not bounded, contrary to what happens in scatterers with finite range potentials at (all but finitely many) fixed energy levels.

As usual in billiards, $T$ preserves a measure $\mu$ that is absolutely continuous to Lebesgue measure, with density

$$
d \mu=\frac{r_{i}}{\Lambda} \cos \varphi^{+} d \theta^{+} d \varphi^{+}, \quad\left(\theta^{+}, \varphi^{+}\right) \in M^{+},
$$

for the normalizing constant $\Lambda=4 \pi \sum_{i=1}^{N} r_{i}$. With respect to this measure, the Sinaĭ billiards is known to be ergodic, mixing and even Bernoulli, [9,21,31]. Our setting is similar enough to conclude mixing (see [12, Section 6.7]), but that doesn't give any quantitative results.

Theorem 1.1 The billiard flow on billiard tables with horns can be modeled as a suspension flow over a Young tower with exponential tails, see Sect. 2.3. The height function $h$ of the suspension has polynomial tails $\mu(\{x \in M: h(x)>t\}) \sim C t^{-\beta}$ for some constant $C>0$ if the horns ${ }^{3}$ are Torricelli trumpets with $\beta>0$ equal to the parameter of the trumpet.

\footnotetext{
${ }^{2}$ Since [18] measures $\Delta \theta$ in incoming angles rather than outgoing as we do, their Theorem 4.2 and 4.3 give the forbidden range $[2-\delta, 2]$.

3 The statistical behavior is governed by the trumpet with the smallest parameter.
} 
It follows that the billiard map has non-zero Lyapunov exponents. This theorem implies exponential mixing rates for the billiard map, and limit laws of the flow w.r.t. the Liouville measure. We state these results later on (Theorem 2.1 and 3.1) as they can be taken from the literature. Although soft-ball billiards will be one of our main tools, the flow on Torricelli trumpets is a flow on a negatively curved surface (in fact, the curvature tends to zero in the cusp). There is extensive literature on such flows, e.g. [14,15,19,29] and references therein to give a sample, but these are predominantly concerned with ergodicity, mixing and Lyapunov exponents. The application to limit laws, and the exact computations of our types of horns (despite similarities to [29, Section 2]) seem to be new.

The next section is concerned with building a Young tower for the billiard map [33], or rather verifying that the methods of Chernov [11] and the soft-ball billiard approach of Bálint $\&$ Tóth [5,6] applies under appropriate conditions. In Sect. 3.1 we then estimate the sojourn times on horn, that lead to the height of the suspension flow of Theorem 1.1.

Notation: We will write $(\theta, \varphi) \in M$ for the position and angle at outgoing collisions, and use $\left(\theta^{+}, \varphi^{+}\right) \in M^{+}$only if we want to emphasize that it is about the outgoing collision. We write $a_{n} \sim b_{n}$ if $\lim _{n} a_{n} / b_{n}=1$ and $a_{n} \approx b_{n}$ if $a_{n} / b_{n}$ have a bounded and positive lim sup and lim inf.

\section{Billiard Maps for Tables with Horns}

\subsection{Conditions to Build a Young Tower}

Young [33] introduced a tower construction and used it (among other things) to prove that the billiard map of the Sinaĭ has exponential decay of correlations. Chernov [11] formulated general conditions under which Young tower with exponential or with polynomial tails for various other billiards besides the Sinai billiard. One is the uniform hyperbolicity of the billiard map, so (despite its discontinuities) with uniform expansion and contraction rates, and the angles between stable and unstable leaves uniformly bounded away from zero. We discuss this for our setting in Sect. 2.2. Additionally distortion has to be controlled, also in order to find a differentiable quotient map (after dividing out the stable direction). Global distortion control is impossible due to grazing collisions (i.e., collisions with $\varphi= \pm \frac{\pi}{2}$ ) at scatterers. Homogeneity strips are therefore introduced in the phase space near $\varphi= \pm \frac{\pi}{2}$ within which distortion control is feasible. This leads, however, to the chopping of unstable leaves and the need for a "growth of unstable manifold" condition in [11, Section 2]. We discuss this for our setting in Sect. 2.3.

In their turn, Bálint \& Tóth give in Definitions 2 and 3 of [5] sufficient conditions in the soft-ball scatterer setting to apply the methods of Chernov. We rely on [5] for the verification of [11, Formulas (26)-(26)]. We summarize these conditions, using their notation, specifically the derivative of the rotation function $\Delta \theta$ :

$$
\kappa(\varphi):=\frac{d}{d \varphi} \Delta \theta(\varphi) \quad \text { for } \varphi=\varphi^{+} .
$$

1. $\inf _{\varphi}|2+\kappa(\varphi)|>0$.

2. $\tau_{\min }>\sup _{\varphi}-2 r_{i} \frac{\kappa(\varphi)}{\omega(\varphi)}$ where $r_{i}$ is the radius of the scatterers and $\omega(\varphi):=\frac{2+\kappa(\varphi)}{\cos \varphi}$.

3. $\Delta \theta$ is piecewise Hölder, i.e., there is $C>0$ and $\alpha \in(0,1)$ such that

$$
\left|\Delta \theta(\varphi)-\Delta \theta\left(\varphi^{\prime}\right)\right| \leq C\left|\varphi-\varphi^{\prime}\right|^{\alpha}
$$


for all second coordinates $\varphi, \varphi^{\prime}$ of points in the same element of a finite partition of the phase space $M^{+}$.

4. $\Delta \theta$ is piecewise $C^{2}$ on the interiors of the partition in the previous item.

5. There is $C>0$ such that $\left|\frac{d \kappa(\varphi)}{d \varphi}\right| \leq C|2+\kappa(\varphi)|^{3}$.

6. There is $\varepsilon>0$ such that $\omega(\varphi)$ from item 2. is monotone on one-sided neighborhoods $\left[\varphi^{*}-\varepsilon, \varphi^{*}\right)$ and $\left(\varphi^{*}, \varphi^{*}+\varepsilon\right]$ of angles $\varphi^{*}$ where $\kappa$ is infinite or discontinuous.

The importance of these conditions is underlined by the fact that hyperbolicity and een ergodicity can fail even if $\kappa=-2$, see [32] where finite range potentials and configurations near grazing collisions are established that lead to homoclinic orbits with nearby elliptic islands.

We list some comments on these properties for Torricelli trumpets, and mention where in this paper they are addressed further.

ad 1 . This condition holds because $\kappa(\varphi)<-2$.

ad 2. This condition holds because $\omega(\varphi)<0$ and $\sup _{\varphi}-2 r_{i} \frac{\kappa(\varphi)}{\omega(\varphi)}=0$. However, if the horns have the shape of pseudo-spheres, which give exponential tails in Theorem 1.1, $\kappa\left( \pm \frac{\pi}{2}\right)=-2$ at grazing collisions and $\sup _{\varphi}-2 r_{i} \frac{\kappa(\varphi)}{\omega(\varphi)}=\infty$. Hence hyperbolicity and even ergodicity when the horns are pseudo-spheres remain unproven.

ad 3. Hölder continuity of the rotation function $\Delta \theta(\varphi)$ holds in our case for $\varphi \approx \pm \frac{\pi}{2}$, but fails near head-on collisions with horns (i.e., $\varphi \approx 0$ ). In fact, if $\varphi=0$, the particle will never leave the horn again. As we will see, $\Delta \theta(\varphi)$ and hence of $\kappa$ are the unbounded. This situation is not covered in [6]; it requires extra arguments (in the shape of adding more "homogeneity strips") to control the distortion. More precisely, we will introduce an equivalent of homogeneity strips, denoted by $\mathbb{I}_{ \pm k}$, which accumulate from both directions on the equator $\{\varphi=0\}$, within which we can control the distortion of $\Delta \theta(\varphi)$ on these $\mathbb{I}_{k}$, see Proposition 2.2. Fortunately, unstable leaves become automatically long, in a way that the need for additional growth lemmas is avoided.

ad 4. The rotation function $\Delta \theta(\varphi)$ is $C^{2}$ on all the intervals of continuity in $\left[-\frac{\pi}{2}, \frac{\pi}{2}\right]$. Because $\Delta \theta(\varphi)$ blows up near $\{\varphi=0\}$, we have to resort to $C^{2}$ smoothness on the (artificial) homogeneity strips $\mathbb{I}_{ \pm k}$ near $\{\varphi=0\}$.

ad 5. This is unproblematic for Torricelli trumpets (see Sects. 3.3).

ad 6. This is unproblematic; the computations in Sect. 3.3 yield this condition automatically.

\subsection{Distortion Control of the Billiard Map}

In this section, we study the distortion of the billiard map $T$. Since the flight map $F$ has bounded distortion inside homogeneity strips (as in [12, Section 5.3], but see Formula (8) below) and the reflection at scatterers goes as for standard billiard maps, we concentrate on the reflection map $R$ for the horns. Here the distortion control when $\cos \varphi \approx 0$ is not an issue, as it can be dealt with in the standard way of introducing homogeneity strips

$$
\mathbb{H}_{ \pm k}=\left\{x \in M^{+}:\left| \pm \frac{\pi}{2}-\varphi^{+}\right| \in\left[(k+1)^{-2}, k^{-2}\right)\right\},
$$

see [12, Section 5.3], and the fact that $\kappa\left(\varphi^{+}\right)$is bounded near $\varphi^{+}= \pm \frac{\pi}{2}$. The additional problem occurs for $\varphi^{+} \approx 0$ because $\Delta \theta$ and hence $\kappa$ are unbounded here. Our solution is as with grazing collisions at scatterers, introduce homogeneity strips within which distortion is controlled. Fortunately, the large expansion in such strips overcomes the artificial chopping in one iterate. Hence the analysis of this case is easier, and doesn't require additional growth 


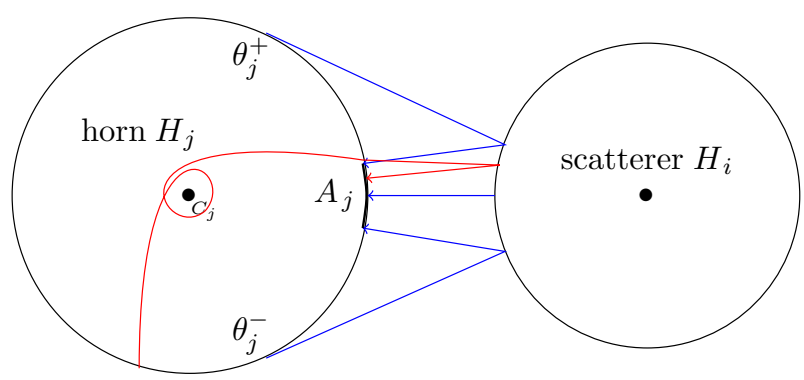

Fig. 1 Trajectories asymptotic to the center $C_{j}$ of $H_{j}$

lemmas. We start this section by describing these homogeneity strips, and then deal with the distortion control, with Proposition 2.2 as main result.

For each horn $H_{j}$ select an other horn or scatterer $H_{i}$, opposite to it.

(i) If $H_{i}$ is a horn, then there is a maximal arc $A_{j} \subset M_{j}^{+}$such that for each $x \in A_{j}$, the trajectory starting at $x$ collides with $H_{i}$ head-on, i.e., $F(x)=\left(\theta_{i}^{-}, 0\right)$, and such a trajectory will not exit $H_{i}$ anymore.

(ii) If $H_{i}$ is a scatterer, then $A_{j}$ is the maximal arc $A_{j} \subset M_{j}^{+}$such that $F(x) \in M_{i}^{-}$and $F \circ T(x)=\left(\theta_{j}^{-}, 0\right) \in M_{j}^{-}$. In other words: trajectories of the flow starting at $x \in A_{j}$ first bounce with $H_{i}$ before head-on colliding with $H_{j}$, and thus not exiting $H_{j}$ again, see Figure 1.

In either case, $A_{j}$ is a smooth curve in $M_{j}^{+}$that stretches across $M_{j}^{+}$in the vertical direction and is transversal to $\left\{\varphi_{j}^{+}=0\right\}$. Let $b_{j}=\left(\theta_{j}^{+},+\frac{\pi}{2}\right)$ and $b_{j}^{\prime}=\left(\theta_{j}^{+},-\frac{\pi}{2}\right)$ be the endpoints of $A_{j}$. The reflection map

$$
R: M_{j}^{-} \backslash\left\{\varphi_{j}^{-}=0\right\} \rightarrow M_{j}^{+} \backslash\left\{\varphi_{j}^{+}=0\right\}, \quad\left(\theta_{j}^{-}, \varphi_{j}^{-}\right) \mapsto\left(\theta^{-}+\Delta \theta\left(-\varphi_{j}^{-}\right),-\varphi_{j}^{-}\right),
$$

is a bijection. The closer to the equator $\mathbb{S}^{1} \times\{0\} \subset M_{j}^{-}$, the stronger the shear of $R$. That is, an $\operatorname{arc}\{\theta\} \times\left(0, \frac{\pi}{2}\right]$ is mapped by $R$ to a spiral curve wrapping infinitely often around the annulus $M_{j}^{+}$, compactifying on $\mathbb{S}^{1} \times\{0\}$ from below, while $\{\theta\} \times\left[-\frac{\pi}{2}, 0\right)$ is mapped by $R$ to a spiral curve wrapping infinitely often around the annulus $M_{j}^{+}$, compactifying on $\mathbb{S}^{1} \times\{0\}$ from above. Conversely, $\Psi_{j}:=R^{-1}\left(A_{j} \backslash\left\{\varphi_{j}^{+}=0\right\}\right)$ consists of two spirals wrapping infinitely often around $M_{j}^{-}$and compactifying on the equator, one from above and one from below. For every point $\left(\theta_{j}^{-}, \varphi_{j}^{-}\right) \in \Psi_{j}$,

(i) if $H_{i}$ is a horn, then $F \circ R\left(\theta_{j}^{-}, \varphi_{j}^{-}\right)$represents a particle head-on colliding with $H_{i}$, so that $T \circ R\left(\theta_{j}^{-}, \varphi_{j}^{-}\right)$is not defined: the particle never leaves $H_{i}$.

(ii) if $H_{i}$ is a scatterer, $T \circ R\left(\theta_{j}^{-}, \varphi_{j}^{-}\right)$represents a particle outgoing from $H_{i}$ and head-on colliding with $H_{j}$, so that $R \circ T \circ R\left(\theta_{j}^{-}, \varphi_{j}^{-}\right)$is not defined: the particle never leaves $H_{j}$ again.

Now $M_{j}^{-} \backslash \overline{\Psi_{j}}$ consists of two strips that wrap around $M_{j}^{-}$infinitely often and approaching $\{\varphi=0\}$ in a spiral fashion from above and below. Let $e_{j}=R^{-1}\left(b_{j}\right), e_{j}^{\prime}=R^{-1}\left(b_{j}^{\prime}\right)$, and let $E_{j}$ be the straight line connecting $e_{j}$ and $e_{j}^{\prime}$ in $M_{j}^{-}$. Then $E_{j}$ cuts $M_{j}^{-} \backslash \overline{\Psi_{j}}$ into infinitely many strips $\mathbb{I}_{ \pm k}, k \in \mathbb{N}$, whose closures are curvilinear rectangles except that they coincide at two opposite corners; note that they wrap around $M_{j}^{-}$once. 


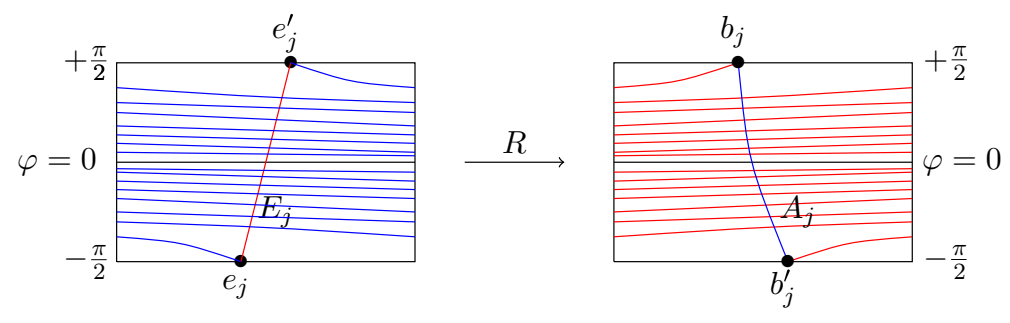

Fig. 2 The curves $E_{j}, \Psi_{j}=R^{-1}\left(A_{j}\right) \subset M_{j}^{-}$and $A_{j}, R\left(E_{j}\right) \subset M_{j}^{+}$

The sets $\mathbb{I}_{ \pm k}$ play the role of homogeneity strips, within which unstable derivatives are uniformly bounded.

Lemma 2.1 Let $\tilde{I}_{ \pm k}$ be the arcs $\mathbb{I}_{ \pm k} \cap\left(\{0\} \times\left[-\frac{\pi}{2}, \frac{\pi}{2}\right]\right)$. Then $\left|\tilde{I}_{ \pm k}\right| \approx k^{-\left(1+\frac{1}{\beta}\right)}=o\left(d\left(\tilde{I}_{k}, 0\right)\right)$ as $k \rightarrow \infty$ for some $\beta>0$, where $d$ is the Euclidean distance on $\left[-\frac{\pi}{2}, \frac{\pi}{2}\right]$.

Proof The precise computation depends on the shape of the horn $H_{j}$, but there is always a leading term of the map $\Delta \theta:\left[-\frac{\pi}{2}, \frac{\pi}{2}\right] \rightarrow \mathbb{R}$ of the form $g: \varphi \mapsto C \varphi^{-\beta}$ for some $0 \neq C \in \mathbb{R}$ and $\beta>0$. Now

$$
\tilde{I}_{k} \sim g^{-1}(2 \pi k, 2 \pi(k+1))=\left(\left(\frac{C}{2 \pi(k+1)}\right)^{1 / \beta},\left(\frac{C}{2 \pi k}\right)^{1 / \beta}\right),
$$

so $\left|\tilde{I}_{k}\right| \sim \frac{1}{\beta k}\left(\frac{C}{2 \pi k}\right)^{1 / \beta} \sim \frac{1}{\beta k} d\left(\tilde{I}_{k}, 0\right)$. The same argument works for $-k$.

Proposition 2.1 Assume that $\kappa\left(\varphi^{+}\right) \leq 0$ on $M^{+}$. Then the stable and unstable leaves $W^{u / s}$ of the billiard map $T$ are uniformly transversal to each other.

Proof In standard hard ball billiards, written in $\varphi_{i}^{+}$and arc-length coordinate $s_{i}$ parametrizing $\partial H_{i}$, with associated coordinates $d \varphi_{i}^{+}$and $d s_{i}$ in the associate tangent space $\mathcal{T} M_{i}$, the cones fields

$$
\tilde{\mathcal{C}}_{\left(s_{i}, \varphi_{i}^{+}\right)}^{u}:=\left\{d s_{i} \cdot d \varphi_{i}^{+} \geq 0\right\} \text { and } \tilde{\mathcal{C}}_{\left(s_{i}, \varphi_{i}^{+}\right)}^{s}:=\left\{d s_{i} \cdot d \varphi_{i}^{+} \leq 0\right\}
$$

serve as unstable and stable cone fields. Indeed, the derivative of the corresponding billiard map from scatterer $i$ to scatterer $j$ is $D \tilde{T}=\left(\begin{array}{cc}1 & 0 \\ 0 & -1\end{array}\right) \cdot D \tilde{F}$, where the derivative of the flight map

$$
D \tilde{F}=\frac{-1}{\cos \varphi_{j}^{+}}\left(\begin{array}{cc}
\frac{\tau}{r_{i}}+\cos \varphi_{i}^{+} & \tau \\
-\frac{\tau}{r_{i} r_{j}}-\frac{\cos \varphi_{j}^{+}}{r_{i}}-\frac{\cos \varphi_{i}^{+}}{r_{j}}-\frac{\tau}{r_{j}}-\cos \varphi_{j}^{+}
\end{array}\right),
$$

in coordinates $\left(s_{i}, \varphi_{i}^{+}\right)$and $\left(s_{j}, \varphi_{j}^{+}\right)$can be easily reconstructed from see [12, Formula (2.26)]. Here $\tau$ is the flight time between $H_{i}$ and $H_{j}$, and we wrote $1 / r_{i}$ and $1 / r_{j}$ for their curvatures. Since all the entries of $D \tilde{T}$ are negative, the above cones are indeed preserved under forward and negative times respectively. The difference with our setting is:

- We use $\theta_{i}^{+}=r_{i} s_{i}$ and $\theta_{i}^{+}=r_{i} s_{i}$ as coordinates. The necessary change of coordinates requires multiplying $D \tilde{F}$ on the left and right with the matrices $\left(\begin{array}{ll}1 & 0 \\ 0 & r_{j}\end{array}\right)$ and $\left(\begin{array}{cc}1 & 0 \\ 0 & 1 / r_{i}\end{array}\right)$, 
respectively. This gives

$$
D F=-\frac{1}{\cos \varphi_{j}^{+}}\left(\begin{array}{cc}
\frac{\tau}{r_{i}}+\cos \varphi_{i}^{+} & \frac{\tau}{r_{i}} \\
-\frac{\tau}{r_{i}}-\frac{r_{j} \cos \varphi_{j}^{+}}{r_{i}}-\cos \varphi_{i}^{+}-\frac{\tau}{r_{i}}-\frac{r_{j} \cos \varphi_{j}^{+}}{r_{i}}
\end{array}\right) .
$$

- The extra shear of the reflection map $R: M_{j}^{-} \rightarrow M_{j}^{+},\left(\theta_{j}^{-}, \varphi_{j}^{-}\right) \mapsto\left(\theta^{-}+\Delta \theta,-\varphi_{j}^{-}\right)$, with derivative $\left(\begin{array}{cc}1 & \kappa \\ 0 & -1\end{array}\right)$ with $\kappa=\frac{d \Delta \theta\left(\varphi^{+}\right)}{d \varphi^{+}}$, instead of the derivative $\left(\begin{array}{cc}1 & 0 \\ 0 & -1\end{array}\right)$ of the reflection map of a standard scatterer.

The resulting derivative is

$$
\begin{aligned}
D T & =\left(\begin{array}{cc}
1 & \kappa \\
0 & -1
\end{array}\right) \cdot D F \\
& =\frac{-1}{\cos \varphi_{j}^{+}}\left(\begin{array}{cc}
\frac{\tau}{r_{i}}+\cos \varphi_{i}^{+}-\frac{\kappa \tau}{r_{i}}-\frac{\kappa r_{j} \cos \varphi_{j}^{+}}{r_{i}}-\kappa \cos \varphi_{i}^{+} & \frac{\tau}{r_{i}}-\frac{\kappa \tau r}{r_{i}}-\frac{\kappa r_{j} \cos \varphi_{j}^{+}}{r_{i}} \\
\frac{\tau}{r_{i}}+\frac{r_{j} \cos \varphi_{j}}{r_{i}}+\cos \varphi_{i}^{+} & \frac{\tau}{r_{i}}+\frac{r_{j} \cos \varphi_{j}^{+}}{r_{i}}
\end{array}\right) .
\end{aligned}
$$

Since $\kappa \leq 0$, again all entries of $D T$ are negative, so

$$
\mathcal{C}_{\left(\theta^{+}, \varphi^{+}\right)}^{u}:=\left\{d \theta^{+} \cdot d \varphi^{+} \geq 0\right\} \text { and } \mathcal{C}_{\left(\theta^{+}, \varphi^{+}\right)}^{s}:=\left\{d \theta^{+} \cdot d \varphi^{+} \leq 0\right\}
$$

are preserved under forward and backward iteration of $D T$, respectively. If $\kappa$ is very negative, then $D T_{\left(\theta^{+}, \varphi^{+}\right)}\left(\mathcal{C}_{\left(\theta^{+}, \varphi^{+}\right)}^{u}\right)$ aligns itself with the horizontal axis. However, the images of the stable cones $D T^{-1}\left(C_{T\left(\theta^{+}, \varphi^{+}\right)}^{s}\right)$ are uniformly compactly contained in $C_{\left(\theta^{+}, \varphi^{+}\right)}^{s}$ because the matrix $D F^{-1}$ is applied after ${ }^{4}$ (the large shear of) $D R^{-1}$. Hence the angle between stable and unstable leaves is uniformly bounded away from zero. This proves the lemma.

The backward singularity sets $\mathcal{S}^{m}:=\cup_{j=0}^{m} T^{-j}\left(\bigcup_{i} \partial H_{i} \times\left\{-\frac{\pi}{2}, \frac{\pi}{2}\right\} \cup \bigcup_{\text {horns }} H_{j} \partial H_{j} \times\{0\}\right)$ for $m \geq 1$, also belong to the stable cone field, and the forward singularity sets $\mathcal{S}^{-m}:=$ $\cup_{j=0}^{m} T^{j}\left(\left(\bigcup_{i} \partial H_{i} \times\left\{-\frac{\pi}{2}, \frac{\pi}{2}\right\} \cup \bigcup_{\text {horns } H_{j}} \partial H_{j} \times\{0\}\right)\right.$ for $m \geq 1$, belong to the unstable cone field.

Proposition 2.2 Let $W=W^{u}$ be an unstable leaf contained in $\mathbb{I}_{ \pm k}$ and bounded away from $\left\{\varphi= \pm \frac{\pi}{2}\right\}$. Then there is $C_{\text {dist }} \in \mathbb{R}$ such that the distortion in the unstable direction

$$
\log \frac{\left|J^{u} R(y)\right|}{\left|J^{u} R(x)\right|} \leq C_{\text {dist }} d_{R(W)}(R(x), R(y)),
$$

where $d_{R(W)}$ indicates arc-length in $R(W)$. In fact, $\log \frac{\left|J^{u} R(y)\right|}{\left|J^{u} R(x)\right|}=o\left(d_{R(W)}(R(x), R(y))\right)$ as $k \rightarrow \infty$, i.e., as $\varphi \rightarrow 0$.

Proof First we assume that $W$ is transversal to horizontal lines in $M$, i.e., transversal to lines of constant $\varphi$. Thus $W$ can be written as the graph of a $C^{1}$-function $w: \tilde{I}_{ \pm k} \rightarrow \mathbb{S}^{1}$. The length-element of arc-length along $W$ is $d s=d s(\varphi)=\sqrt{1+\left(w^{\prime}(\varphi)^{2}\right)} d \varphi$. Recall that $R(\theta, \varphi)=(\theta+\Delta \theta(\varphi),-\varphi)$ is the reflection map, and $\Delta \theta^{\prime}(\varphi)=\kappa(\varphi)$. Then the image of $d s$ under $R$ is

$$
d R(s)=\sqrt{d \varphi^{2}+\left(w^{\prime}(\varphi)+\kappa(\varphi)\right)^{2} d \varphi^{2}}=\sqrt{1+\left(w^{\prime}(\varphi)+\kappa(\varphi)\right)^{2}} d \varphi .
$$

\footnotetext{
4 If we had used a billiard map $T: M^{-} \rightarrow M^{-}$in terms of incoming coordinates, it would have been the unstable image cones rather than the stable image cones, that would have been uniformly compactly contained in the unstable cone fields.
} 
Transversality of $W$ means that $\left|w^{\prime}\right|$ is bounded, so

$$
\frac{d R(\varphi)}{d \varphi}=\sqrt{1+\left(w^{\prime}(\varphi)+\kappa(\varphi)\right)^{2}} \approx \kappa(\varphi) \approx C \beta \varphi^{-(\beta+1)} .
$$

where we used that leading term of $\kappa$ is $C \beta \varphi^{-(\beta+1)}$ as in Lemma 2.1. Hence, taking $x=$ $(w(\varphi), \varphi)$ and $y=(w(\varphi+\varepsilon), \varphi+\varepsilon)$, the distortion

$$
\log \frac{\left|J^{u} R(y)\right|}{\left|J^{u} R(x)\right|} \approx-(\beta+1) \log \left(1+\frac{\varepsilon}{\varphi}\right) \approx \frac{-\varepsilon(1+\beta)}{\varphi} .
$$

Now to find $d_{R(W)}(R(y), R(x))$ we integrate $d R(s)$ over $[\varphi, \varphi+\varepsilon]$. This gives

$$
\begin{aligned}
\int_{\varphi}^{\varphi+\varepsilon} \sqrt{1+\left(w^{\prime}(v)+\kappa(v)\right)^{2}} d v & \approx \int_{\varphi}^{\varphi+\varepsilon}|\kappa(v)| d v \\
& =|\Delta \theta(\varphi+\varepsilon)-\Delta \theta(\varphi)| \approx C\left(\varphi^{-\beta}-(\varphi+\varepsilon)^{-\beta}\right) \\
& =C \varphi^{-\beta}\left(1-\left(1+\frac{\varepsilon}{\varphi}\right)^{-\beta}\right) \sim \beta C \varepsilon \varphi^{-(1+\beta)},
\end{aligned}
$$

where in the last approximation we used that $|\varepsilon|$ is small compared to $|\varphi|$ as shown in Lemma 2.1. Formula (7) follows.

For $\varphi \rightarrow 0$, we get $1+\sup \left|w^{\prime}(\varphi)\right|^{2}=o(\kappa(\varphi))$, so that the $\approx$ becomes $\sim$ in this case, and the extra factor $\varphi^{-\beta}$ accounts for the little $o$.

The flight map $F$ has similar distortion properties as (7), as can be derived from the distortion result in [12, Lemma 5.27], except that the exponent on the right-hand side is $1 /(1+1 / \beta)$, where $1+1 / \beta$ is the exponent in the width in the strips $\left|\mathbb{I}_{ \pm k}\right| \approx k^{1+1 / \beta}$, see [12, Formulas (5.8), (5.21) and Lemma 5.27] and the adaptation for general exponents in e.g. [16, Lemma 3.1]. Because of Lemma 2.1 for $\mathbb{I}_{ \pm k}$ and the usual width of the strips $\mathbb{H}_{ \pm k}$, this exponent becomes $\min \left\{\frac{1}{3}, \frac{\beta}{\beta+1}\right\}$.

Because the uniform expansion of the billiard map $T=R \circ F$, we get the following distortion estimate for the billiard map:

$$
\log \frac{\left|J^{u} T^{n}(y)\right|}{\left|J^{u} T^{n}(x)\right|} \leq C_{\text {dist }} d_{T^{n}(W)}\left(T^{n}(x), T^{n}(y)\right)^{\min \left\{\frac{1}{3}, \frac{\beta}{\beta+1}\right\}},
$$

for a potentially large uniform constant $C_{\text {dist }}$ and all $n \geq 1$ and $x, y$ in the same unstable leaf of $T^{n}$. The absolute continuity of holonomies in [11, Formula (2.3)] is a corollary of (8), see [10,34]. Together with [12, Theorem 5.2 and Section 5.7] this give sufficient distortion control to conclude that the quotient tower map $T_{\bar{\Delta}}$ presented in the next section, has a Hölder derivative.

\subsection{Building a Young Tower with Exponential Tails}

A Young tower [33] is a schematic dynamical system, in fact an extension over a dynamical system $(X, T)$, of the form $\left(\Delta, T_{\Delta}, \mu_{\Delta}\right)$, where the space

$$
\Delta=\bigsqcup_{i}^{\sigma_{i}-1} \bigsqcup_{\ell=0} \Delta_{i, \ell}
$$


The sets $\Delta_{i, \ell}$ are copies of the $\Delta_{i, 0}$ and the tower map $T_{\Delta}$ acts as

$$
T_{\Delta}: x \in \Delta_{i, \ell} \mapsto \begin{cases}x \in \Delta_{i, \ell+1} & \text { if } 0 \leq \ell<\sigma_{i}-1 ; \\ T^{\sigma(x)}(x) \in \Delta_{0}:=\sqcup_{i} \Delta_{i, 0} & \text { if } \ell=\sigma_{i}-1,\end{cases}
$$

and $\left(\Delta, T_{\Delta}\right)$ factors over $(X, T)$ via $\pi: \Delta \rightarrow X, \pi(u, \ell)=T^{\ell}(u)$ for $\left.(u, \ell) \in \Delta_{i, \ell}\right)$ ). The return map $T^{\sigma_{i}}: \Delta_{0} \rightarrow \Delta_{0}$ to the base $\Delta_{0}=\sqcup \Delta_{i, 0}$ is a uniformly hyperbolic map with certain distortion properties, preserving an SRB-measure $\mu_{0}$. Here $\sigma: \Delta_{0} \rightarrow \mathbb{N}$ with $\sigma_{i}:=\left.\sigma\right|_{\Delta_{i, 0}}$ constant for all $i$ is called the roof function. We speak of exponential tails if there is $\lambda \in(0,1)$ such that $\mu_{0}(\{x: \sigma(x)>n\})=O\left(\lambda^{n}\right)$. We can extend $\mu_{0}$ to an $T_{\Delta}$-invariant measure by setting $\left.\mu_{\Delta}\right|_{\Delta_{i, \ell}}=\left.\bar{\sigma}^{-1} \mu_{0}\right|_{\Delta_{i, 0}}$ for normalizing constant $\bar{\sigma}=$ $\sum_{n \geq 1} n \mu_{0}(\{\sigma(x)=n\})$. This measure $\mu_{\Delta}$ pushes down to a $T$-invariant SRB-measure on $(X, T)$ via $\mu=\mu_{\Delta} \circ \pi^{-1}$. The existence of a Young tower with exponential tails implies that the underlying system $(X, T, \mu)$ is exponentially mixing (provided $\left.\operatorname{gcd}\left(\sigma_{i}: i \in \mathbb{N}\right\}=1\right)$ and satisfies the Central Limit Theorem for Hölder observables, see [33]. A step in the argument is to consider the quotient tower $\left(\bar{\Delta}, T_{\bar{\Delta}}\right)$ obtained by collapsing stable leaves to points. The smoothness (Gibbs-Markov) of the quotient map $T_{\bar{\Delta}}^{\sigma_{i}}$, as described in e.g. [33, Section 3.1] and [12, Theorem 5.2 and Section 5.7] relies on the distortion estimates given in Section 2.2, specifically Formula (8).

Chernov [11, Theorem 2.1] proved a general theorem on the existence of a Young tower with exponential tails for non-uniformly hyperbolic invertible maps, based on a set of conditions concerning expansion and distortion control along unstable leaves and specific "growth of unstable manifolds" conditions (2.6)-(2.8) in [11]. He continues to verify these conditions for various billiard systems, of which the standard Sinal billiard maps ${ }^{5}$ is the most relevant to us, see [11, Sections $6 \&$ 7]. Bálint \& Tóth verify these conditions for soft scatterers, expressed as Definition $2 \& 3$ in [6]. In the previous sections we verified most of the Chernov resp. Bálint \& Tóth conditions, and here we combine these steps to the final verification. That is, we indicate which adaptations in the arguments of [11, Section 7] are still required.

Chernov [11, Section 7] uses two metrics to obtain hyperbolic expansion:

- The $p$-(pseudo-)metric which has the best expansion properties, but only that after a close-to-grazing collision with corresponding cut into homogeneity strips, the expansion has a one iterate delay.

- The Euclidean metric. Now the expansion factor in unstable directions occurs instantaneously at collisions, but it is not always $\geq 1$. Therefore a particular iterate $T^{m}$ of the billiard map $T$ is chosen, which multiplies the number of discontinuity curves $\mathcal{S}^{m-1}$ and $\cup_{n=0}^{m-1} T^{-n}\left(\cup_{j} \partial H_{j} \times\{0\}\right)$, and corresponding boundaries of homogeneity strips $\cup_{k \geq k_{0}} \cup_{n=0}^{m-1} T^{-n}\left(\partial \mathbb{I}_{ \pm k}\right)$.

However, combining the two metrics, one can prove uniform expansion (contraction) of unstable (stable) leaves, see [11, Lemma 7.1].

Let $W$ be any unstable leave of length $\leq \delta_{0}$. It may be cut into at most $K_{m}+1$ pieces by $\mathcal{S}^{m-1}$, where $K_{m}$ depends only on $m$ and the number of scatterers and horns. In the next $m$ iterate, it may be cut again, even into countably many pieces, by curves in $\cup_{n=0}^{m-1} T^{-n}(\{\varphi=$ 0 at horns $\left.\left.\} \cup \bigcup_{k \geq k_{0}} \partial \mathbb{I}_{ \pm k}\right) \cup\left\{\varphi= \pm \frac{\pi}{2}\right\} \cup \bigcup_{k \geq k_{0}} \partial \mathbb{H}_{ \pm k}\right)$. We label these pieces as $W_{k_{1}, \ldots, k_{m}, j}$, where $1 \leq j \leq K_{m}+1, k_{i} \in \mathbb{Z}$ and $T^{m-n}\left(W_{k_{1}, \ldots, k_{m}, j}\right) \subset \mathbb{I}_{k_{n}}$. Bear in mind that some of these labels can refer to the empty set. Head-on collisions with horns and grazing collisions

5 i.e., disjoint strictly convex fully elastic scatterers with $C^{3}$ boundaries on a compact flat table, first fully treated in [33]. 
have their own homogeneity strips $\mathbb{I}_{ \pm k}$ and $\mathbb{H}_{ \pm k}$ where the expansion of the billiard map is $\approx k^{1+\beta}$ and $\approx k^{2}$ respectively; we will use $v:=\min \{2,1+\beta\}$ for the worst case of the two. The unstable expansion for $T_{1}=T^{m}$ on a piece $W_{k_{1}, \ldots, k_{m}}$ of unstable manifold thus becomes

$$
\left|J_{1}^{u}(x)\right| \geq L_{k_{1}, \ldots, k_{m}}:=\max \left\{\Lambda_{1}, \frac{1}{C_{\exp }} \prod_{k_{i} \neq 0} k_{i}^{v}\right\} .
$$

This product $\prod_{k_{i} \neq 0}$ then reappears in the definition ${ }^{6}$ of $\Theta:=2 \sum_{k \geq k_{0}} k^{-v} \leq \frac{7}{v} k_{0}^{1-v}$. We need to choose $k_{0}$ so large that, as in [11, Formula (7.5)] with corresponding constant $B_{0}$,

$$
\left(K_{m}+1\right)\left(\Lambda_{1}^{-1}+2 B_{0} \Theta\right)<1 .
$$

Also [11, Lemma 7.2] needs to be adjusted to:

Lemma 2.2 For all $\delta>0$, there is $B=B(m)$ such that

$$
\sum_{k_{1}, \ldots, k_{m} \geq 2} \min \left\{\delta,\left(\kappa_{1} \cdots k_{m}\right)^{-v}\right\}<B(m) \delta^{\frac{v-1}{2 m}} .
$$

But the proof goes as in [11, Appendix], with some minor and obvious adaptations.

Thus we can apply Chernov's main theorem for the billiard map, which we restate here:

Theorem 2.1 For any type of horn discussed in this paper, for every $\alpha \in(0,1)$ there is $\lambda \in(0,1)$ such that the billiard map $(M, T)$ has exponential decay of correlations:

$$
\left|\int_{M} v \cdot w \circ T^{n} d \mu-\int_{M} v d \mu \int_{M} w d \mu\right|=O\left(\lambda^{n}\right)
$$

for the SRB-measure $\mu$ and $\alpha$-Hölder functions $v, w: M \rightarrow \mathbb{R}$ and also the Central Limit Theorem holds for $v$ provided it is not cohomologous to a constant function.

\section{The Billiard Flow}

The billiard flow can now be modeled as a suspension flow over this Young tower, i.e., the space is now $\Delta^{h}:=\sqcup_{i, \ell} \Delta_{i, \ell} \times[0, h(x)] / \sim$ where $(x, h(x)) \sim\left(T_{\Delta}(x), 0\right)$, and the flow $\phi_{\Delta}^{t}(x, u)=(x, u+t) \in \Delta^{h}$. The height function $h$ is either equal to the (bounded) flight time $\tau(x)$ between a horn/scatterer and a scatterer, or equal to the flight time $\tau(x)$ between a scatterer and a horn plus the sojourn time $2 t_{\max }$ in the horn. The $\phi_{\Delta}^{t}$-invariant measure $\mu_{\Delta}^{h}=\bar{h}^{-1} \mu_{\Delta} \otimes$ Leb for the normalizing constant $\bar{h}=\int_{\Delta} h(x) d \mu_{\Delta}$ or $\bar{h}=1$ if this integral is infinite, because in this infinite measure case, there is no normalization. The corresponding flow-invariant measure $\mu^{h}$ is the push-down $\mu_{\Delta}^{h} \circ \pi_{h}^{-1}$ where $\pi_{h}(x, u)=\phi^{u} \circ \pi(x)$.

The computations in Sect. 3.3 show that the tails of $h$ have the asymptotics

$$
\mu\left(\left\{x \in M_{i}^{+}: h \circ F(x)>t\right\}\right)=\frac{r_{i}}{\Lambda} \int_{\left\{x \in M_{i}^{+}: \tau(x)+2 t_{\max } \circ F(x)>t\right\}} \cos \varphi d \mu \sim C t^{-\beta}
$$

for some constant depending only on the shape of the horns. In fact, the exponent $\beta$ is equal to the parameter $\beta$ of the Torricelli trumpet, and therefore $\mu^{h}$ is finite if and only if $\beta>1$.

Due to Theorems 1.1 and 2.1 with (10) we can apply results from [28] or [8] to derive the following distributional limit theorems for the flow.

6 This $\Theta$ is called $\theta_{0}$ in [11]. 
Theorem 3.1 Consider a Sină billiard with Torricelli trumpets as horns, where $\beta>1$ is the smallest parameter of these trumpets. Let $v$ be a measurable bounded ${ }^{7}$ Hölder observable such that $\inf _{x \in H_{i}}|v(x)|>0$ for at least one horn $H_{i}$ with parameter $\beta$. Then:

- If $\beta \in(1,2)$, then $v$ satisfies a Stable Law:

$$
\frac{1}{T^{1 / \beta}}\left(\int_{0}^{T} v \circ \phi^{t} d t-T \int v d \mu^{h}\right) \Rightarrow^{d} G_{\beta} \quad \text { as } T \rightarrow \infty .
$$

- If $\beta=2$, then $v$ satisfies a non-Gaussian Central Limit Theorem:

$$
\frac{1}{\sqrt{T \log T}}\left(\int_{0}^{T} v \circ \phi^{t} d t-T \int v d \mu^{h}\right) \Rightarrow^{d} \mathcal{N}(0,1) \quad \text { as } T \rightarrow \infty .
$$

- If either $\beta>1$ and $\operatorname{supp}(v)$ is compact (rather than containing a horn), or $\beta>2$, then $v$ satisfies a standard Central Limit Theorem: provided $v$ is not cohomologous to a constant function, there is a constant $\sigma>0$ such that

$$
\frac{1}{\sigma \sqrt{T}}\left(\int_{0}^{T} v \circ \phi^{t} d t-T \int v d \mu^{h}\right) \Rightarrow^{d} \mathcal{N}(0,1) \quad \text { as } T \rightarrow \infty .
$$

\subsection{Dynamics of the Flow on Horns}

Let $H$ be a surface of revolution in $\mathbb{R}^{3}$ obtained by revolving the curve $x=x(z)$ around the $z$-axis. We will use the radius $r=r(z)=\sqrt{x^{2}+y^{2}}$ as radius of $H$ and $z=z(r)$ is the inverse function. Thus $H$ has the parametrization

$$
\sigma(z, \theta)=(r(z) \cos \theta, r(z) \sin \theta, z), \quad z \geq z_{0}, \theta \in[0,2 \pi) .
$$

Abbreviate $r_{0}:=r\left(z_{0}\right)$.

Example 3.1 The area and volume of $H$ are

$$
A:=2 \pi \int_{z_{0}}^{\infty}\left(1+r^{\prime 2}\right) r d z \quad \text { and } \quad V:=\pi^{2} \int_{z_{0}}^{\infty} r^{2} d z
$$

respectively. For $r(z)=z^{-\beta}$, we get $A=\infty, V=\frac{\pi}{2 \beta-1}<\infty$ (painter's paradox) for $\beta \in\left(\frac{1}{2}, 1\right]$. This holds specifically for the case $\beta=1$, i.e., $r=1 / z$, when $H$ is called the trumpet of Torricelli (or also Gabriel's horn). For $\beta>1$ we have $A, V<\infty$ and for $\beta \leq \frac{1}{2}$, both $A, V=\infty$. The Gaussian curvature of such a surface

$$
\kappa_{G}=-\frac{r^{\prime \prime}}{r\left(1+r^{\prime 2}\right)^{2}}=-\frac{\beta^{2}+\beta}{z^{2}\left(1+\beta^{2} z^{-2(1+\beta)}\right)^{2}} \rightarrow 0 \quad \text { as } z \rightarrow \infty .
$$

For the next exposition, see [2, Section $4 \mathrm{C}]$. A geodesic $\Gamma$ is the path on $H$ traced out by a unit mass particle moving along $H$ at unit speed with no external forces other than the holonomic constraints keeping it on $H$. Assume the geodesic starts at $\left(\theta_{0}, z_{0}\right) \in \partial H$, making an incoming angle $\varphi_{0} \in\left[-\frac{\pi}{2}, \frac{\pi}{2}\right]$ with the inward vertical meridian. Since $\varphi^{ \pm}$are angles with the outward normal vector to $\partial H$, we have $\varphi_{0}=-\varphi^{-}=\varphi^{+}$, so that $\kappa\left(\varphi_{0}\right)=\kappa\left(\varphi^{+}\right)$ as used in Section 2.1. The kinetic energy

$$
E_{k i n}=\frac{1}{2}|v|^{2}=\frac{1}{2}\left(\left(1+r^{\prime 2}\right) \dot{z}^{2}+r^{2} \dot{\theta}^{2}\right)=\frac{1}{2}
$$

\footnotetext{
7 Note that the space is not compact, so Hölder continuity doesn't imply boundedness.
} 
is one constant of motion. Due to the rotational symmetry (using Noether's Theorem), the $z$-component of the angular momentum

$$
r^{2} \dot{\theta}=r_{0}|v| \sin \varphi_{0}=r_{0} \sin \varphi_{0}
$$

is the second constant of motion. Inserting $r \dot{\theta}=|v| \sin \varphi$ (where $\varphi=\varphi(z)$ is the angle with the inward vertical meridian) we get a derived constant of motion (Clairaut's Theorem)

$$
r(z) \sin \varphi(z)=r_{0} \sin \varphi_{0}
$$

The absolute value of $\sin \varphi$ is largest at the highest point of the geodesic (where $\sin \varphi_{\max }=1$, $r_{\min }=r_{0} \sin \varphi_{0}, \theta=\theta_{\max }$ and $\left.z=z_{\max }=z\left(r_{\min }\right)\right)$, then the geodesic spirals down again (symmetrically to the upwards spiral), until it hits $\partial H$ at an angle $-\varphi_{0}$ with the inward vertical meridian.

The question we pose ourselves is:

What is the time $t_{\max }$ needed of the geodesic particle to reach the top at $z_{\max }$ ?

This has a direct consequence for the tails of geodesic flow if these sojourns inside the horns are modeled by suspension flow with height function $2 t_{\max }$ and base map $R: M_{j}^{-} \rightarrow M_{j}^{+}$ as in (2).

\subsection{Computation of $t_{\max }$ and $\theta_{\max }$}

From (12) combined with (13) we find

$$
\dot{z}=\frac{d z}{d t}=\sqrt{\frac{1-r(z)^{2} \dot{\theta}^{2}}{1+r^{\prime}(z)^{2}}}=\sqrt{\frac{1-r_{0}^{2} r(z)^{-2} \sin ^{2} \varphi_{0}}{1+r^{\prime}(z)^{2}}} .
$$

Therefore

$$
d t=\sqrt{\frac{1+r^{\prime}(z)^{2}}{1-r_{0}^{2} r(z)^{-2} \sin ^{2} \varphi_{0}}} d z
$$

and

$$
t_{\max }=\int_{0}^{t_{\max }} d t=\int_{z_{0}}^{z_{\max }} \sqrt{\frac{1+r^{\prime}(z)^{2}}{1-r_{0}^{2} r(z)^{-2} \sin ^{2} \varphi_{0}}} d z .
$$

Using the change of coordinates $u=\frac{r_{0}}{r(z)}\left|\sin \varphi_{0}\right|$, so $z(r(u))=z\left(\frac{r_{0}\left|\sin \varphi_{0}\right|}{u}\right), z=z_{0} \Leftrightarrow u=$ $\left|\sin \varphi_{0}\right|, z=z_{\max } \Leftrightarrow u=1$ and $d z=-\frac{r_{0}\left|\sin \varphi_{0}\right|}{u^{2}} \frac{1}{r^{\prime}\left(z\left(\frac{r_{0}\left|\sin \varphi_{0}\right|}{u}\right)\right)} d u$, we find ${ }^{8}$

$$
t_{\max }=r_{0}\left|\sin \varphi_{0}\right| \int_{\left|\sin \varphi_{0}\right|}^{1} \frac{1}{u^{2}} \cdot \sqrt{\frac{1+r^{\prime}\left(z\left(\frac{r_{0}\left|\sin \varphi_{0}\right|}{u}\right)\right)^{-2}}{1-u^{2}}} d u .
$$

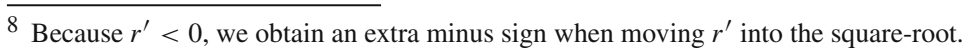


Now for the difference between the entrance position $\theta_{0}$ and the position $\theta_{\max }$ reached at the top of the geodesic, we find, using (13) and the previous computation for $\dot{z}$ :

$$
\begin{aligned}
\theta_{\max }-\theta_{0} & =\int_{\theta_{0}}^{\theta_{\max }} d \theta=\int_{0}^{t_{\max }} \dot{\theta} d t=\int_{0}^{t_{\max }} \frac{r_{0} \sin \varphi_{0}}{r^{2}} d t \\
& =\int_{z_{0}}^{z_{\max }} \frac{r_{0} \sin \varphi_{0}}{r^{2}} \frac{1}{\dot{z}} d z \\
& =r_{0} \sin \varphi_{0} \int_{z_{0}}^{z_{\max }} \frac{1}{r^{2}} \sqrt{\frac{1+r^{\prime}(z)^{2}}{1-r_{0}^{2} r(z)^{-2} \sin ^{2} \varphi_{0}}} d z .
\end{aligned}
$$

This should be compared to Formula (5.2) in [5] expressing $\Delta \theta$ in terms of the potential of a soft scatterer. Applying the transformation $u=\frac{r_{0}}{r(z)}\left|\sin \varphi_{0}\right|$ as before, we get

$$
\theta_{\max }-\theta_{0}=\operatorname{sgn}\left(\varphi_{0}\right) \int_{\left|\sin \varphi_{0}\right|}^{1} \sqrt{\frac{1+r^{\prime}\left(z\left(\frac{r_{0}\left|\sin \varphi_{0}\right|}{u}\right)\right)^{-2}}{1-u^{2}}} d u
$$

Throughout (and following the notation of $[5,6]$ ) we let

$$
\Delta \theta=2\left(\theta_{\max }-\theta_{0}\right)=2 \operatorname{sgn}\left(\varphi_{0}\right) \int_{\left|\sin \varphi_{0}\right|}^{1} \sqrt{\frac{1+r^{\prime}\left(z\left(\frac{r_{0}\left|\sin \varphi_{0}\right|}{u}\right)\right)^{-2}}{1-u^{2}}} d u .
$$

be the difference in incoming and outgoing angle of the obstacle as function of angle of incidence $\varphi_{0}$. Its derivative w.r.t. $\varphi_{0}$ is

$$
\begin{aligned}
\kappa\left(\varphi_{0}\right)= & \frac{\partial \Delta \theta\left(\varphi_{0}\right)}{\partial \varphi_{0}}=-2 \sqrt{1+\left(r^{\prime}\left(z_{0}\right)\right)^{-2}}+2 r_{0} \cos \varphi_{0} \times \\
& \int_{\left|\sin \varphi_{0}\right|}^{1} \frac{\left.z^{\prime} \frac{r_{0}\left|\sin \varphi_{0}\right|}{u}\right)}{\sqrt{1+r^{\prime}\left(z\left(\frac{r_{0}\left|\sin \varphi_{0}\right|}{u}\right)\right)^{2}}} \frac{r^{\prime \prime}\left(z\left(\frac{r_{0}\left|\sin \varphi_{0}\right|}{u}\right)\right)}{r^{\prime}\left(z\left(\frac{r_{0}\left|\sin \varphi_{0}\right|}{u}\right)\right)^{2}} \frac{1}{u \sqrt{1-u^{2}}} d u .
\end{aligned}
$$

For convex obstacles, i.e., with $z^{\prime}<0$ and $r^{\prime \prime}>0$, the two terms in this expression have the same sign. The first term $<-2$, whereas the second varies between 0 (as $\varphi_{0} \rightarrow \pm \pi / 2$ ) and potentially $-\infty$ (as $\varphi_{0} \rightarrow 0^{\mp}$ ). Therefore, unless $r^{\prime}\left(z_{0}\right)=-\infty$ (as would be the case for a pseudo-sphere) $\kappa\left(\varphi_{0}\right)$ is bounded way from $[-2,0]$ as required in [6] to obtain uniform hyperbolicity.

\subsection{Torricelli's Trumpets}

Assume that the horn is the surface of revolution of the curve $r(z)=z^{-\beta}$, with $r^{\prime}(z)=$ $-\beta z^{-(1+\beta)}, r^{\prime \prime}(z)=\beta(1+\beta) z^{-(2+\beta)}$ and $z(r)=r^{-1 / \beta}$. Inserting the equations for $r(z)$ into (15) gives $r^{\prime}\left(\frac{r_{0}\left|\sin \varphi_{0}\right|}{u}\right)=\beta^{-2} z\left(\frac{r_{0}\left|\sin \varphi_{0}\right|}{u}\right)^{2(1+\beta)}=\beta^{-2} z\left(\frac{u}{r_{0}\left|\sin \varphi_{0}\right|}\right)^{2(1+\beta) / \beta}$ and

$$
t_{\max }=\left|\sin \varphi_{0}\right|^{-\frac{1}{\beta}} \underbrace{\beta^{-1} r_{0}^{-\frac{1}{\beta}} \int_{\left|\sin \varphi_{0}\right|}^{1} \frac{1}{u^{2}} \cdot \sqrt{\frac{\beta^{2}\left(r_{0}\left|\sin \varphi_{0}\right|\right)^{\frac{2(1+\beta)}{\beta}}+u^{\frac{2(1+\beta)}{\beta}}}{1-u^{2}}} d u}_{I\left(\varphi_{0}\right)} .
$$


The integral $I\left(\varphi_{0}\right)$ tends to a positive constant $I_{0}=\beta^{-1} r_{0}^{-\frac{1}{\beta}} \int_{0}^{\frac{\pi}{2}}(\sin \alpha)^{\frac{\beta-1}{\beta}} d \alpha$ as $\varphi_{0} \rightarrow 0$, so the leading asymptotics of $t_{\max }$ is $\left|\sin \varphi_{0}\right|^{-1 / \beta} I_{0}$. This gives tails on the height function,

$$
\mu\left(\left(\theta, \varphi_{0}\right): 2 t_{\max }>t\right)=2 \pi \mu\left(\left|\sin \varphi_{0}\right|<\left(\frac{t}{2 r_{0} I\left(\varphi_{0}\right)}\right)^{-\beta}\right) \sim 4 \pi\left(\frac{t}{2 r_{0} I_{0}}\right)^{-\beta} .
$$

Applying the same formulas to (16), we get

$$
\Delta \theta\left(\varphi_{0}\right)=\frac{2 \operatorname{sgn}\left(\varphi_{0}\right)}{\left|\sin \varphi_{0}\right|^{\frac{1+\beta}{\beta}}} \underbrace{\frac{1}{\beta r_{0}^{\frac{1+\beta}{\beta}}} \int_{\left|\sin \varphi_{0}\right|}^{1} \sqrt{\frac{\beta^{2}\left(r_{0}\left|\sin \varphi_{0}\right|\right)^{\frac{2(1+\beta)}{\beta}}+u^{\frac{2(1+\beta)}{\beta}}}{1-u^{2}}} d u}_{J\left(\varphi_{0}\right)},
$$

and $J\left(\varphi_{0}\right) \rightarrow \beta^{-1} r_{0}^{-\frac{1+\beta}{\beta}} \int_{0}^{\frac{\pi}{2}}(\sin \alpha)^{\frac{1+\beta}{\beta}} d \alpha$ as $\varphi_{0} \rightarrow 0$. Therefore the reflection map $R$ : $M_{j}^{-} \rightarrow M_{j}^{+}$becomes

$$
R:\left(\theta^{-}, \varphi^{-}\right) \mapsto\left(\theta^{-}+2 \operatorname{sgn}\left(\varphi_{0}\right)\left|\sin \varphi_{0}\right|^{-\frac{1+\beta}{\beta}} J\left(\varphi_{0}\right),-\varphi^{-}\right), \quad \varphi_{0}=-\varphi^{-} .
$$

Since $J\left(\varphi_{0}\right) \rightarrow 0$ as $\varphi_{0} \rightarrow \pm \frac{\pi}{2}$ we get $R\left(\theta, \varphi^{-}\right) \rightarrow\left(\theta, \mp \frac{\pi}{2}\right)$ as $\varphi^{-} \rightarrow \pm \frac{\pi}{2}$. Inserting the above into (18), we find

$$
\begin{aligned}
\kappa\left(\varphi_{0}\right)= & -2 \operatorname{sgn}\left(\varphi_{0}\right) \sqrt{1+\beta^{-2} r_{0}^{-2(1+\beta) / \beta}}-\frac{1+\beta}{\beta^{2}} \frac{2 \operatorname{sgn}\left(\varphi_{0}\right)\left|\sec \varphi_{0}\right|}{\left(r_{0}\left|\sin \varphi_{0}\right|\right)^{(1+\beta) / \beta}} \times \\
& \int_{\left|\sin \varphi_{0}\right|}^{1} \frac{1}{\sqrt{u^{2(1+\beta) / \beta}+\beta^{2}\left(r_{0}\left|\sin \varphi_{0}\right|\right)^{2(1+\beta) / \beta}}} \frac{u^{2(1+\beta) / \beta}}{\sqrt{1-u^{2}}} d u .
\end{aligned}
$$

As $\varphi_{0}$ increases from 0 to $\pi / 2, \kappa\left(\varphi_{0}\right)$ increases from $-\infty$ to $-2 \sqrt{1+\beta^{-2} r_{0}^{-2(1+\beta) / \beta}}$, and it is smooth with a finite limit as $\varphi_{0} \rightarrow \pm \frac{\pi}{2}$, giving the Hölderness of $\Delta \theta$ away from $\varphi_{0}=0$. Also $\kappa\left(\left[-\frac{\pi}{2}, \frac{\pi}{2}\right]\right) \cap[-2,0]=\emptyset$, so that hyperbolicity is guaranteed.

The leading term of $\kappa\left(\varphi_{0}\right)$ is $C\left|\varphi_{0}\right|^{-\frac{1+2 \beta}{\beta}}$ for some $C>0$, so, since $\kappa$ is a smooth function of $\varphi_{0} \neq 0$, the leading term of $\kappa^{\prime}\left(\varphi_{0}\right)$ in absolute value is

$$
\frac{1+2 \beta}{\beta} C\left|\varphi_{0}\right|^{-\frac{1+3 \beta}{\beta}} \leq C\left|\varphi_{0}\right|^{-3 \frac{1+2 \beta}{\beta}}=O\left(\left|2+\kappa\left(\varphi_{0}\right)\right|^{3}\right) \quad \text { as } \varphi_{0} \rightarrow 0,
$$

whenever $\beta>-2 / 3$. Hence, for every $\beta>0$, item 5. in Section 2.1 holds. By the same token, recalling that $\omega\left(\varphi_{0}\right)=\frac{2+\kappa\left(\varphi_{0}\right)}{\cos \varphi_{0}}$ (see item 2. in Section 2.1),

$$
\omega^{\prime}\left(\varphi_{0}\right):=\frac{\kappa^{\prime}\left(\varphi_{0}\right)+\left(2+\kappa\left(\varphi_{0}\right)\right) \tan \varphi_{0}}{\cos \varphi_{0}} \text { is bounded away from } 0,
$$

for $\varphi_{0}$ close to 0 . Therefore $\omega\left(\varphi_{0}\right)$ is monotone in one-sided neighborhoods of $\left\{\varphi_{0}=0\right\}$, and item 6. in Section 2.1 holds.

Acknowledgements HB gratefully acknowledges the support of FWF grant P31950-N45 and Stiftung AÖU Project 103öu6. He also wants to thank Péter Bálint for his explanations of his and Chernov's papers [5,6,11], Ian Melbourne for his input on the literature on limit laws, and Homero Canales for verifying some of the lengthier computations in this paper. Thanks also to the referees for their vigilance and many helpful remarks.

Funding Open access funding provided by University of Vienna. 
Open Access This article is licensed under a Creative Commons Attribution 4.0 International License, which permits use, sharing, adaptation, distribution and reproduction in any medium or format, as long as you give appropriate credit to the original author(s) and the source, provide a link to the Creative Commons licence, and indicate if changes were made. The images or other third party material in this article are included in the article's Creative Commons licence, unless indicated otherwise in a credit line to the material. If material is not included in the article's Creative Commons licence and your intended use is not permitted by statutory regulation or exceeds the permitted use, you will need to obtain permission directly from the copyright holder. To view a copy of this licence, visit http://creativecommons.org/licenses/by/4.0/.

\section{References}

1. Aaronson, J., Denker, M.: The Poincaré series of $\mathbb{C} \backslash \mathbb{Z}$. Ergod. Theor. Dyn. Syst. 19, 1-20 (1999)

2. Arnol'd, V.I.: Mathematical Methods of Celestial Mechanics, 4th edn. Springer, New York (1984)

3. Baldwin, P.: Soft billiard systems. Physica D 29, 321-342 (1988)

4. Bálint, P., Butterley, O., Melbourne, I.: Polynomial decay of correlations for flows, including Lorentz gas examples. Commun. Math. Phys. 368, 55-111 (2019)

5. Bálint, P., Tóth, P.I.: Correlation decay in certain soft billiards. Commun. Math. Phys. 243, 55-91 (2003)

6. Bálint, P., Tóth, P.I.: Mixing and its rate in soft and hard billiards motivated by the Lorentz process. Physica D 187, 128-135 (2004)

7. Bruin, H., Terhesiu, D.: Regular variation and rates of mixing for infinite measure preserving almost Anosov diffeomorphisms. Ergod. Theor. Dyn. Syst. 40, 663-698 (2020)

8. Bruin, H., Terhesiu, D., Todd, M.: Pressure function and limit theorems for almost Anosov flows. Commun. Math. Phys. 382, 1-47 (2021)

9. Bunimovič, L., Sinaŭ, Y.: The fundamental theorem of the theory of scattering billiards. Mat. Sb. (N.S.) 90, 415-431 (1973), 479

10. Bunimovič, L., Sină̌, Y., Chernov, N., Statistical properties of two-dimensional hyperbolic billiards, Uspekhi Mat. Nauk, 46, : 43-92 (in Russian). Russian Mathematical Surveys 46(1991), 47-106 (1991). ((in English))

11. Chernov, N.: Decay of correlations and dispersive billiards. J. Stat. Phys. 94, 513-556 (1999)

12. Chernov, N., Markarian, R.: Chaotic Billiards, Mathematical Surveys and Monographs, 127. Amer. Math. Soc, Providence, RI (2006)

13. Chernov, N., Zhang, H.-K.: A family of chaotic billiards with variable mixing rates. Stoch. Dyn. 5, 535-553 (2005)

14. Coudène, Y.: Sur le mélange du flot géodésique, Monogr. Enseign. Math., 43, Enseignement Math., Geneva, 13-24 (2013)

15. Coudène, Y., Schapira, B.: Generic measures for geodesic flows on nonpositively curved manifolds. J. Ec. Polytech. Math. 1, 387-408 (2014)

16. Demers, M., Zhang, H.-K.: Spectral analysis of hyperbolic systems with singularities. Nonlinearity $\mathbf{2 7}$, 379-433 (2014)

17. Donnay, V.: Elliptic islands in generalized Sinai billiards. Ergodic Theory Dyn. Syst. 16, 975-1010 (1996)

18. Donnay, V., Liverani, C.: Potential on the two-torus for which the Hamiltonian flow is ergodic. Commun. Math. Phys. 135, 267-302 (1991)

19. Donnay, V., Pugh, C.: Anosov geodesic flows for embedded surfaces, Geometric methods in dynamics. II. Astérisque 287, 61-69 (2003)

20. Eslami, P., Liverani, C.: Mixing rates for symplectic almost Anosov maps, Prepint 2020, arXiv:2003.07551

21. Gallavotti, G., Ornstein, D.: Billiards and Bernoulli schemes. Commun. Math. Phys. 38, 83-101 (1974)

22. Hu, H.: Conditions for the existence of SBR measures of "almost Anosov" diffeomorphisms. Trans. Amer. Math. Soc. 352, 2331-2367 (2000)

23. Hu, H., Zhang, X.: Polynomial decay of correlations for almost Anosov diffeomorphisms. Ergod. Theor. Dyn. Syst. 39, 832-864 (2019)

24. Knauf, A.: Ergodic and topological properties of Coulombic periodic potentials. Commun. Math. Phys. 100, 89-112 (1987)

25. Knauf, A.: On soft billiard systems. Physica D 36, 259-262 (1989)

26. Kubo, I.: Perturbed billiard systems, I. The ergodicity of motion of a particle in a compound central field. Nagoya Math. J. 61, 1-51 (1975)

27. Liverani, C., Martens, M.: Convergence to equilibrium for intermittent symplectic maps. Commun. Math. Phys. 260, 527-556 (2005) 
28. Melbourne, I., Varandas, P.: Convergence to a Lévy process in the Skorohod $\mathcal{M}_{1}$ and $\mathcal{M}_{2}$ topologies for nonuniformly hyperbolic systems, including billiards with cusps. Commun. Math. Phys. 375, 653-678 (2020)

29. Pollicott, M., Weiss, H.: Ergodicity of the geodesic flow on non-complete negatively curved surfaces. Asian J. Math. 13, 405-419 (2009)

30. Sinal̆, Y.: On the foundations of the ergodic hypothesis for dynamical systems of statistical mechanics. Dok. Akad. Nauk SSSR 153, 1261-1264 (1963)

31. Sinal̆, Y.: Dynamical systems with elastic reflections: Ergodic properties of dispersing billiards. Russ. Math. Surv. 25, 137-189 (1970)

32. Turaev, D., Rom-Kedar, V.: Elliptic islands appearing in near-ergodic flows. Nonlinearity 11, 575-600 (1998)

33. Young, L.-S.: Statistical properties of dynamical systems with some hyperbolicity. Ann. Math. 147, 585$650(1998)$

34. Young, L.-S.: Recurrence times and rates of mixing. Israel J. Math. 110, 153-188 (1999)

Publisher's Note Springer Nature remains neutral with regard to jurisdictional claims in published maps and institutional affiliations. 